\title{
NEOPLASIAS PRIMÁRIAS MÚLTIPLAS EM PACIENTES COM CÂNCER COLORRETAL
}

\author{
Marcelo de Souza CURY* e Nora Manoukian FORONES**
}

RESUMO - Introdução - A presença de uma segunda neoplasia maligna, tendo sitio e histologia diferentes da primeira, define o conceito de neoplasias primárias múltiplas. Com o aumento da sobrevida dos pacientes com câncer, houve um aumento de neoplasias primárias múltiplas. Este estudo objetiva avaliar a freqüência de neoplasias primarias múltiplas em pacientes com diagnóstico de câncer colorretal do Setor de Oncologia da Disciplina de Gastroenterologia da Universidade Federal de São Paulo. Pacientes e Métodos - No periodo de 1993 a 1998, 145 pacientes com câncer colorretal foram acompanhados no Setor de Oncologia da Disciplina de Gastroenterologia da Universidade Federal de São Paulo. Destes, cinco pacientes (3,4\%) tinham neoplasias primárias múltiplas. O segundo tumor foi confirmado por exame histológico, sendo excluidas as possibilidades de metástase. Resultados - $A$ idade média dos doentes com neoplasias primárias múltiplas foi de 60,6 anos, quatro eram do sexo feminino e um do masculino, três apresentaram doença no reto, um no cólon direito e um no cólon esquerdo. Os outros sítios foram mama, útero, útero e vagina, pele e lábio. Um paciente foi a óbito e os outros estão em acompanhamento, sendo que dois, há mais de três anos. Dois pacientes tinham sido submetidos a radioterapia pélvica antes do aparecimento da neoplasia colorretal. Em um paciente o tumor colorretal antecedeu o outro câncer e em quatro apareceu posteriormente ao diagnóstico da outra neoplasia primária. Discussão - A prevalência de neoplasias primárias múltiplas foi de 3,4\%, sendo maior no sexo feminino. Câncer de endométrio foi o outro tumor maligno mais freqüente. Quarenta por cento dos nossos pacientes foram submetidos a radioterapia. Acreditamos ser importante o acompanhamento dos pacientes para o diagnóstico precoce de uma nova neoplasia em qualquer paciente que já tenha apresentado um câncer.

DESCRITORES - Neoplasias primárias múltiplas. Neoplasias colorretais. Fatores de risco.

\section{INTRODUÇÃO}

O aumento da sobrevida dos doentes com tumores malignos é um dos principais fatores responsáveis pela incidência crescente de neoplasias primárias múltiplas ${ }^{(3,8,10)}$. Outros fatores relacionados são: hereditariedade e efeito carcinogênico da terapia empregada $a^{(3)}$.
O termo neoplasia primária múltipla (NPM) é definido como o aparecimento de uma segunda neoplasia com histologia e sítio diferentes da primeira. Os critérios de WARREM in IKEDA et al. ${ }^{(14)}$ determinam que devem ser malignas, comprovadas por histologia, estarem em locais anatomicamente distintos e excluir a possibilidade de que a segunda seja metástase da primeira.

Setor de Oncologia, Disciplina de Gastroenterologia, Escola Paulista de Medicina, Universidade Federal de São Paulo.

* Médico Residente.

** Professora Adjunta.

Endereço para correspondência: Dra. Nora Manoukian Forones - Rua Botucatu, 740 - $2^{\circ}$. andar - 04023-900 - São Paulo, SP. e-mail: nora@gastro.epm.br 
Sua importância reside na possibilidade de preparar-se estratégias para diagnóstico precoce e terapêutica curativa em pacientes identificados como tendo risco aumentado.

O câncer colorretal é um dos tumores mais freqüentes em humanos, acometendo 60,8 homens e 42,3 mulheres a cada 100.000 habitantes no período de 1985 a 1989 segundo COHEN et al. ${ }^{(2)}$, sendo responsável por $15 \%$ dos tumores malignos nos Estados Unidos da América ${ }^{(4)}$. No Brasil, dados do Ministério da Saúde estimam uma incidência de 19.050 novos casos para o ano de 1999, sendo a quarta causa de câncer entre os homens e a quinta entre as mulheres ${ }^{(1)}$.

Este estudo tem por objetivo avaliar a freqüência de neoplasias primárias múltiplas em pacientes com diagnóstico de câncer colorretal do Setor de Oncologia da Disciplina de Gastroenterologia da Universidade Federal de São Paulo (UNIFESP-EPM).

\section{CASUÍSTICA E MÉTODOS}

Foram estudados, retrospectivamente, 145 doentes com adenocarcinoma colorretal acompanhados pelo Setor de Oncologia da Disciplina de Gastroenterologia da UNIFESP-EPM, no período de 1993 a 1998. Os pacientes com câncer colorretal foram avaliados pela possibilidade de apresentar outra neoplasia primária já diagnosticada ou que tenha se desenvolvido durante o acompanhamento.

Foram incluídos no grupo de neoplasias primárias múltiplas (NPM) os pacientes com diagnóstico comprovado por exame anatomopatológico e que preenchessem os seguintes critérios:

- neoplasias malignas, comprovadas histologicamente,

- excluir a possibilidade de serem metástases,

- estarem em locais distintos.

Foram analisadas características dos doentes com o diagnóstico de NPM, como relação entre as neoplasias, idade, sexo, história familiar, localização colônica e extracolônica e evolução dos pacientes.

\section{RESULTADOS}

Entre os 145 pacientes tratados por câncer colorretal, no período de 93 a 98, cinco pacientes (3,4\%) apresentaram NPM. Quatro eram do sexo feminino e um masculino, a idade variou entre 55 e 68 anos (média 60,6 e mediana 61) (Tabelas 1, 2). O estadiamento da neoplasia colônica é apresentado na Tabela 1. Quatro pacientes apresentaram uma neoplasia extracolônica e um apresentou duas neoplasias extracolônicas. Dois pacientes foram submetidos a radioterapia pélvica antes de desenvolver o câncer de reto.

Em três pacientes o tumor extracolônico foi o primeiro. Os locais de acometimento foram: mama, útero, útero e vagina, lábio e pele. As regiões do cólon acometidas foram: três no reto, um no cólon direito e um no cólon esquerdo.

A diferença de tempo entre o aparecimento dos tumores variou de um a seis anos, com média de quatro anos. A sobrevida após o diagnóstico do tumor de cólon variou de 1 a 5 anos (média de 2,6 anos). Atualmente, quatro doentes estão em acompanhamento e um evoluiu para óbito 1 ano após o diagnóstico da doença colônica. Apenas um paciente tinha história familiar, apresentando um irmão com neoplasia de cólon, outro de reto e o pai com neoplasia de estômago.

TABELA 1 - Idade e sexo dos pacientes com NPM estádio clínico do câncer colorretal

\begin{tabular}{cccc}
\hline $\mathbf{N}^{\mathbf{0}}$ & Idade & Sexo & Estádio \\
\hline 1 & 57 & $\mathrm{~F}$ & III \\
2 & 61 & $\mathrm{M}$ & III \\
3 & 55 & $\mathrm{~F}$ & I \\
4 & 68 & $\mathrm{~F}$ & II \\
5 & 62 & $\mathrm{~F}$ & III \\
\hline
\end{tabular}

TABELA 2 - Localização, tipo histológico da primeira e segunda neoplasia e do intervalo entre ambas

\begin{tabular}{llllll}
\hline $\mathbf{N}$ & \multicolumn{2}{c}{$\mathbf{1}^{\mathbf{a}}$ neoplasia } & $\begin{array}{c}\text { Intervalo } \\
\text { (anos) }\end{array}$ & & \multicolumn{2}{c}{$\mathbf{2}^{\text {a }}$ neoplasia } \\
\hline & Local & Histologia & & Local & Histologia \\
\hline 1 & Mama & Adenoca & 6 & Cólon D & Adenoca \\
2 & Pele & Basocelular & 1 & Reto & Adenoca \\
3 & Cólon E & Adenoca & 2 & Útero & Adenoca \\
& & & & Lábio & CEC \\
5 & Reto & Adenoca & 1.5 & Reto & Adenoca \\
\end{tabular}

$\mathrm{CEC}=$ Carcinoma espinocelular; Adenoca $=$ Adenocarcinoma;

Cólon $\mathrm{D}$ = cólon direito; Cólon $\mathrm{E}=$ cólon esquerdo 


\section{DISCUSSÃO}

A prevalência estimada de neoplasias primárias múltiplas varia muito em cada série, pois não existe padronização na forma das pesquisas, critérios de inclusão e de registro. Desde a primeira descrição até hoje, aumentaram muito as publicações sobre este tema; um dos motivos seria o melhor e mais cuidadoso acompanhamento dos pacientes, e o outro, o aumento de sua incidência ${ }^{(3,8,15)}$.

Entre as causas de NPM e o aumento de sua incidência, estão o aumento da sobrevida, fatores hereditários, efeito carcinogênico e imunossupressor da terapia empregada, e a fatores de risco a que o paciente esteve exposto (por exemplo: álcool, tabaco) ${ }^{(3,5,8,15)}$.

Os locais já descritos de segunda neoplasia em pacientes com câncer colorretal, por ordem de freqüência foram: útero, colo de útero, mama, estômago, bexiga, próstata, rim, pulmão, intestino delgado, fígado, faringe e pele ${ }^{(8)}$. Neoplasia de útero foi a mais freqüente associação, assim como o sexo feminino.

Neoplasias primárias múltiplas ocorreram em $0,62 \%$ de crianças com neoplasias malignas nos arquivos de registro de câncer da Alemanha ${ }^{(8)}$ entre 1980 e 1995 . O risco relativo de uma segunda neoplasia, em crianças é de 3,6 nos países do norte europeu, de 5,8 no Reino Unido, e de 10,8 na Itália ${ }^{(8)}$.

WARREN in IKEDA et al. ${ }^{(14)}$ em 1932 descreveram uma chance de 1,8 a $11 \%$ de uma segunda neoplasia em adultos. MOERTEL et al. ${ }^{(9)}$, em 1961, descreveram um risco de 5,1 de aparecer uma segunda neoplasia primária em 3.700 pacientes adultos. IKEDA et al. ${ }^{(6)}$ estudaram 199 pacientes com câncer de cólon e 477 com câncer gástrico e encontraram 13 pacientes com ambas (oito sincrônicos e cinco metacrônicos). FRÖDIN et al. ${ }^{(3)}$ estimaram que $9 \%$ a $12 \%$ dos pacientes com neoplasia na Suécia, terão uma segunda neoplasia no decorrer de suas vidas, se sobreviverem à primeira neoplasia. TEPPO et al. ${ }^{(12)}$ estimam que em adultos com câncer de cólon, existe um risco de 1.36 de desenvolver um neoplasia de mama e de 1.78 de neoplasia de útero.

No presente estudo, 3,4\% dos pacientes com câncer colorretal apresentaram uma segunda neoplasia. Acredita-se que este número possa ser subestimado devido ao diagnóstico tardio que ocorre freqüentemente no Brasil, quando comparado a outros centros. Possivelmente alguns dos doentes em acompanhamento irão desenvolver uma segunda neoplasia, se sobreviverem à neoplasia em acompanhamento.

O reto foi o segmento colônico mais acometido. A diferença temporal entre o aparecimento das neoplasias foi em média de quatro anos (variando de um a seis). O sexo feminino foi o mais acometido. Este dado não apresenta consenso na literatura, variando muito em cada série. TAKITA et al. ${ }^{(11)}$ encontraram 12,1:1 (homens : mulheres) ao estudar NPM em pacientes com câncer oral; já KEGEL e SCHMIEDER $^{(7)}$ encontraram duas mulheres para cada homem, estudando NPM em indivíduos submetidos a radioterapia.

A evolução destes pacientes foi melhor que a média geral, fato este que pode ser explicado pelo acompanhamento freqüente dos doentes com câncer, o que torna o diagnóstico de uma segunda neoplasia mais precoce. No presente estudo, 60\% apresentaram linfonodos comprometidos. Nos registros de câncer da Itália, VERCELLI et al. ${ }^{(13)}$ encontraram melhor prognóstico nas mulheres em todos os grupos.

Este estudo sugere que o acompanhamento após o diagnóstico e tratamento de uma primeira neoplasia, deve ser criterioso para o risco de uma segunda neoplasia, sobretudo em mulheres e em pacientes que foram submetidos a tratamento adjuvante como quimio e radioterapia, principalmente radioterapia pélvica.

Cury M de S, Forones NM. Multiple primary neoplasms in colorectal cancer patients. Arq Gastroenterol, São Paulo, 2000;37(2):89-92.

ABSTRACT - Introduction - Multiple primary neoplasms are defined as a second malignance having histology and site different from the first. The increase of the life expectation in cancer patients leads to an increase in multiple primary neoplasms incidence. This study analyzes the characteristics of patients with colorectal cancer and another primary neoplasm. Patients and Methods - In the period from 1993 to 1998, 145 patients with colorectal cancer were accompanied in the Oncology Division of Gastroenterology of Federal University of São Paulo, São Paulo, SP, Brazil. Five patients (3.4\%) had multiple primary neoplasms. The possibility of metastasis were excluded and the second cancer was confirmed by hystological examination. Results - The medium age was of 60.6 years old, four were female and one male. Three had rectum cancer and two colon cancer, one in the right colon and one in the left colon. The other site of cancer was breast, uterus, uterus and vagina, skin and lip. One patient died and the others were in attendance, two for more than three years. Two patients received pelvic radiotherapy before the rectal cancer. In one patient the tumor colorectal cancer appeared before the other cancer, and in four it appeared later on to the diagnosis of the other primary neoplasia. Discussion - The prevalence of multiple primary neoplasms was of 3,4\%, being major in female. Uterus' cancer was the more frequent association. Radiotherapy was performed in $40 \%$ of patients. We believe that attendance of cancer patients is very important to precocious diagnosis and treatment of multiple primary neoplasms.

HEADINGS - Neoplasms, multiple primary. Colorectal neoplasms. Risk factors 


\section{REFERÊNCIAS BIBLIOGRÁFICAS}

1. Brasil. Ministério da Saúde. Instituto Nacional do Câncer. Estimativa de Incidência e Mortalidade de Câncer para 1999 (http://www.inco.org.br/).

2. Cohen AM, Minisky BD, Schilska, RL. Cancer of the colon. In: DeVito VT, Hellman S, Rosmberg AS, editor. Cancer. Principles and practice of oncology. 5. ed. New York: Lippincott Raven; 1997. p.1144-96.

3. Frödin JE, Ericsson J, Barlow L. Multiple primary malignant tumors in a National Cancer Registry. Acta Oncol 1997;36:465.

4. Ganz RC. Câncer de cólon. In: Murad AM, editor. Oncologia-Bases clínicas do tratamento. Rio de Janeiro: Guanabara Koogan; 1996. p.176-9.

5. Hawkins MM. Multiple primary cancers in population - based cancer registries. Eur J Cancer 1996;32:1279.

6. Ikeda Y, Mori M, Kajiyama K, Haraguchi Y, Sugimachi K. Multiple primary gastric and colorectal cancer in Japan. Int Surg 1995;80:37.

7. Kegel W, Schmieder A. The problem of multiple cancers. Rontgenblatter 1982;35:411.

8. Mitchell ME, Johnson JA III, Wilton PB. Five primary sinchronous neoplasms of the gastrointestinal tract. J Clin Gastroenterol 1996;34:284.
9. Moertel CG, Dockerty MB, Banggenstoss AH. Multiple primary malignant neoplasms. Cancer 1961;14:221.

10. Queiroz LACP, Falcão JB, Forones NM. Neoplasias primárias extraintestinais em pacientes com câncer colorretal. Rev Bras Clin Terap 1996;22:57.

11. Takita M, Taniguchi B, Lin M, Machiya T, Kawamoto T, Okunishi Y, Sakuda M. Multiple malignant tumors combined with oral cancer from annual of the pathological autopsy cases in Japan, 1984-1988. Osaka Daigaku Zasshi 1990;35:365.

12. Teppo L, Pukkala E, Saxén E. Multiple cancer - an epidemiologic exercise in Finlandia. J Natl Cancer Inst 1985;75:207.

13. Vercelli M, Quaglia A, Casella C, Mangone L. Cancer patient survival in the elderly in Italy. ITACARE Working Group. Tumori 1997;83:490.

14. Warren in Ikeda Y, Mori M, Kajiyama K, Haraguchi Y, Sugimachi K. Multiple primary gastric and colorectal cancer in Japan. Int Surg 1995;80:37.

15. Westermeir T, Kaatsch P, Schoetzau A, Michaelis J. Multiple primary neoplasms in childhood: data from the German children's cancer registry. Eur J Cancer, $1998 ; 34: 687$.

Recebido para publicação em 26/4/1999. Aprovado para publicação em 20/12/1999. 